\title{
The Essence and Origin of the Magnetic Constant
}

\author{
Nader Butto \\ Rabin Medical Center, Dgania, Petah-Tikva, Israel \\ Email: nader.butto@gmail.com
}

How to cite this paper: Butto, N. (2020) The Essence and Origin of the Magnetic Constant. Journal of High Energy Physics, Gravitation and Cosmology, 6, 663-670. https://doi.org/10.4236/jhepgc.2020.64045

Received: August 19, 2020

Accepted: September 26, 2020

Published: September 29, 2020

Copyright $\odot 2020$ by author(s) and Scientific Research Publishing Inc. This work is licensed under the Creative Commons Attribution International License (CC BY 4.0).

http://creativecommons.org/licenses/by/4.0/

\begin{abstract}
In this study, the essence and origin of the magnetic constant are discussed and a mechanism that allows real estimations of the magnetic constant based upon the vacuum density description is proposed. By considering the vacuum as a liquid with a measurable density and the electron as a vortex, hydrodynamic laws are applied to measure the diminished momentum of a rotating electron in a vacuum, thus obtaining a value similar to the experimentally derived value of the magnetic constant. A consequence of this description is that the magnetic constant can be expressed as the shear stress per unit time of the vacuum; this means that it is an observable vacuum parameter and not a fundamental constant.
\end{abstract}

\section{Keywords}

Magnetic Constant, Magnetic Permeability, Shear Stress, Vacuum Density, Vacuum Viscosity

\section{Introduction}

Physical constants are physical quantities that are generally believed to be both universal in nature and constant over time. It is generally assumed that the values of fundamental constants such as the magnetic constant $\mu_{o}$ electric constant $\varepsilon_{0}$, and speed of light in a vacuum $c$ are the same throughout space-time potentially because they depend on the vacuum's superfluid characteristics.

Universal but dimensioned physical constants such as these are generally referred to as fundamental physical constants. Regardless of the theoretical status of these quantities, the fact that they are constant (independent of both the time and position of the measurement) must necessarily be verified experimentally.

In previous articles [1], the nature and the origin of the fine structure constant was described. Furthermore, new mechanism and analytical formula for understanding the gravity constant $G$ was presented [2]. 
In this article magnetic constant essence and origin are discussed.

To date, no theory has been able to explain the real essence or precise origin of the magnetic constant or magnetic permeability although its name implies that it expresses the permeability of the vacuum and the degree to which it allows magnetic fields to expand. The magnetic constant can be defined as a fundamental invariant quantity and is one of three components of Maxwell's equations that define free space. The classical behavior of the electromagnetic field is described by these equations, which predict that the speed $c$ with which electromagnetic waves (such as light) propagate through the vacuum is related to the electric constant $\varepsilon_{0}$ and magnetic constant $\mu_{0}$ as follows [3]:

$$
c=\frac{1}{\sqrt{\varepsilon_{0} \mu_{0}}}
$$

This equation implies that the speed at which electromagnetic radiation propagates depends on the permittivity and permeability of the vacuum, which are widely considered to be absolute fundamental constants that are invariant in the vacuum.

The magnetic field produced by an electric current or a moving electric charge in a vacuum gives rise to the magnetic permeability. In classical electromagnetic theory, free space resists the formation or propagation of magnetic field-induced photons. In classical physics, free space, which is sometimes referred to as the vacuum of free space, corresponds to a theoretically perfect vacuum and is considered to be a reference medium [4] [5].

In a classical vacuum, $\mu_{0}$ has a precisely defined value, namely [6] [7]

$$
\mu_{0}=4 \pi \times 10^{-7} \mathrm{~N} / \mathrm{A}^{2} \approx 1.2566370614 \cdots \times 10^{-6} \mathrm{~m} \cdot \mathrm{kg} / \mathrm{A}^{2} \cdot \mathrm{s}^{2} .
$$

This paper discusses the essence and origin of the magnetic constant $\mu_{0}$, and its dependence on the density of the vacuum. Its value is obtained by applying hydrodynamic laws and calculating the shear stress of the vacuum.

\section{The Nature of the Vacuum}

Several widely accepted theories, such as special relativity, relativistic quantum field theory, and superfluid vacuum theory, indicate that the vacuum is a fluid with a well-known density. However, its microscopic structure is still largely unknown despite being a subject of intense studies in the field of superfluid vacuum theory.

Maxwell's equations combine electrical and magnetic effects and mathematically quantify their interactions. They were explicitly developed as fluid dynamical models; thus, they require an underlying physical medium. In deriving these equations, Maxwell made certain assumptions about the nature of the medium that carries electromagnetic waves. The primary assumption was that it could be described using the perfect fluid vortex theory developed by Hemlholtz [8].

Likewise, relativistic quantum field theory also assumes the physical vacuum to be a non-trivial medium with a certain associated energy because the concept of absolutely empty space contradicts the postulates of quantum mechanics. Ac- 
cording to quantum field theory, pairs of virtual particles are continually being created and annihilated even in the absence of real particles.

In previous article [9], a new theory of gravitation was presented, according to which the gravitation force is a pull force due to vortex formation of the vacuum. The vortex curves the vacuum (space-time) around it, attract and condense energy and dust to its center to form the mass. The gradient pressure in the vortex creates a flow that upon interaction with an object transfers a part of its momentum to the object and pushes it toward the center.

Superfluid vacuum theory [10] [11] [12] [13] describes the physical vacuum as a quantum superfluid that behaves like a frictionless fluid with extremely high thermal conductivity. It is a perfect fluid in the sense that it is nonparticulate and has no structural memory, i.e., once changed, it has no tendency to return to its former condition. The theory also proposes a mass generation mechanism that may replace or supplement the electroweak Higgs theory. It has been shown that masses of elementary particles could arise from their interaction with the superfluid vacuum, which is similar to the gap generation mechanism in superconductors [14] [15].

Vacuum energy density is generally viewed as a fundamental property of the universe, with a magnitude that is independent of the method used to assess its value, i.e., whether it is subatomic, astronomical, or cosmological. Although there is no consensus as to its value, the most generally accepted estimate relies mainly on general relativity and is based on astronomical observations that determine the curvature of space-time and the expansion of the universe. The universe's expansion can be measured based on the following relationship between a galaxy's velocity $v$ and its distance $d[16]$ :

$$
v=H_{0} d,
$$

where $H_{0}$ is Hubble's constant. This is known as Hubble's Law and is based on the idea that the universe is constantly expanding; thus, galaxies are receding from each other at a constant speed per unit distance. Thus, more distant objects move more rapidly than nearby ones.

It is important to note that studies into the universe's expansion rate have shown that its average density is close to the critical density in which gravitational attraction will eventually balance this expansion and halt it. In his theory of general relativity, Einstein demonstrated that matter causes the surrounding space to curve, thus giving rise to gravity. Both the overall geometry and fate of the universe are controlled by the density of the matter within it.

The universe's density $\rho$ is typically expressed as a fraction of the critical density $\rho_{\text {cr }}$ via $\Omega=\rho / \rho_{\text {cr. If }} \Omega$ is less than 1 (known as an open universe), the universe's final fate will be a cold death" where gravity cannot stop its expansion and it expands forever, albeit at an ever-decreasing rate. If $\Omega$ is greater than 1 (known as a closed universe), gravity eventually halts the universe's expansion and causes it to recollapse. Finally, if $\Omega$ is exactly equal to 1 (known as a flat universe), the universe's density is equal to the critical density and expansion will halt only after an infinite time. 
Currently, summing the estimated contributions to the total density parameter $\Omega_{0}$ gives $1.02 \pm 0.02$, thus indicating that the universe's density is close to critical. In the standard cosmological model, the critical density (which defines the boundary between an open and closed universe) is represented as follows [17]:

$$
\rho_{\text {cr }}=\frac{3 H_{0}^{2}}{8 \pi G}=1.88\left(h^{2}\right) \times 10^{-29} \mathrm{~g} / \mathrm{cm}^{3},
$$

where $H_{0}=71 \mathrm{~km} / \mathrm{s} / \mathrm{Mpc}, G$ is Newton's gravitational constant, and $\mathrm{h} \equiv \mathrm{H} / 71$ $\mathrm{km} / \mathrm{sec} \cdot \mathrm{Mpc}$. The most recent result [18] estimated the cosmological density $\rho$ to be $11.11( \pm 1.05) 10^{-27} \mathrm{~kg} / \mathrm{m}^{3}$.

Considering that the inertial mass of the observable universe $M_{u}$ is as follows:

$$
M_{u}=\frac{c^{3}}{2 H_{0} G}=0.8720532288 \times 10^{56} \mathrm{~kg},
$$

and that its volume $V_{u}$, given its observed radius $R_{u}$, is

$$
V_{u}=\frac{4 \pi R_{u}^{3}}{3}=\frac{4 \pi c^{3}}{3 H_{0}^{3}}=8.9364367479 \times 10^{81} \mathrm{~m}^{3},
$$

the cosmological density can be estimated as follows:

$$
\frac{M_{u}}{V_{u}}=9.75839983 \times 10^{-27} \mathrm{~kg} / \mathrm{m}^{3} .
$$

The best estimate of the cosmological density is that it is between 9.75839983 $\times 10^{-27}$ and $11.11( \pm 1.05) \times 10^{-27} \mathrm{~kg} / \mathrm{m}^{3}$.

\section{Magnetic Constant and Shear Stress}

It is commonly accepted that quantum waves can travel in an ideal vacuum, thus implying that an ideal vacuum behaves like both a liquid and a gas. Thus, the vacuum energy has real observable physical consequences and its properties can be observed through these physical effects [19] [20].

This paper proposes the magnetic permeability as an equivalent to the shear stress per unit area of the vacuum, which depends on the vacuum density. Thus, the shear stress of the vacuum will now be calculated to demonstrate that it is equivalent to the magnetic permeability (constant).

Magnetic permeability and viscosity have much in common. The magnetic permeability of free space is defined as the amount of resistance encountered while forming a magnetic field in a classical vacuum. As stated above, its value is defined as $\mu_{0}=4 \pi \times 10^{-7} \mathrm{~N} \cdot \mathrm{A}^{-2}[21]$.

Viscosity is the degree to which a fluid opposes relative motion between two of its surfaces that are moving at different velocities. Therefore, a fluid's viscosity is often referred to as its thickness and is a measure of its resistance to gradual deformation by shear or tensile stress.

While considering the vacuum as a fluid, its viscous resistance is related to the virtual particles present. These particles represent aspects of the fields they are used to describe, and the speed of light in a vacuum is an intrinsic property of the electromagnetic field. 
The specific resistance of a conductor can be thought of as the diminished momentum of the electrons passing along the conductor's length. Thus, electrical resistance $R$ has units of Ohms, which is defined as $\mathrm{kg} \cdot \mathrm{m} / \mathrm{s}$, per $\mathrm{m}$ of conductor.

The idea that electrons can be considered as vortices was first proposed by Helmholtz in 1858 [22], and treated mathematically by Maxwell, who applied his theory of molecular vortices to magnetic phenomena [23]. According to Helmholtz's theory, particles are vortically moving ring-shaped masses of a homogeneous, incompressible, frictionless fluid. Vortices, namely portions of fluid in rotational motion, will always exist out of the same portion of fluid and are immune to dissipation, i.e., will continue to exist forever.

In previous article [24], a new theory is proposed in which the electron has a structure and a shape. The electron is a frictionless vortex with conserved momentum made out of virtual photons that acquire mass when moving in the vortex at the speed of light. The vortex shape electron allowed to resolve the enigmatic wave-particle duality [25].

According to the vortex theory of the electron, an electron is a vortex rotating around its axis, experiences some resistance owing to interaction with the adjacent vacuum. Its diminished momentum $P$ is determined by dividing the momentum, which given by the vacuum density $\rho$ and vortex angular velocity $\bar{u}$, by the vortex circumference $\lambda$ :

$$
P=\frac{\rho \bar{u}}{\lambda},
$$

where $\bar{u}=c$, i.e., $3 \times 10^{8} \mathrm{~m} / \mathrm{s}$, and the vacuum density $\rho=9.7583993 \times 10^{-27}$ $\mathrm{kg} / \mathrm{m}^{3}$. The vortex circumference $\lambda=2 \pi r$, where $r$ is its radius. If $r$ is considered as the Compton radius of the electron, $3.86 \times 10^{-13} \mathrm{~m}$, then the electron vortex circumference is $2.4263102367(11) \times 10^{-12} \mathrm{~m}$, which is the CODATA 2014 value for the electron's Compton wavelength [26].

Using these values, the electron's diminished momentum can be calculated as follows:

$$
P=\frac{\rho c}{\lambda}=1.206572 \times 10^{-6} \mathrm{~N} \cdot \mathrm{s} / \mathrm{A}^{2},
$$

which is of the same order of magnitude as the magnetic constant $\mu_{0}$. However, the units in Equation (8) express the shear stress and are different from those for the magnetic constant.

Momentum has units of $\mathrm{kg} \cdot \mathrm{m} / \mathrm{s}$. Linear momentum is a conserved quantity; this means that the linear momentum of a closed system (i.e., one that is not affected by external forces) cannot change. Momentum can equivalently be expressed in Newton seconds (N.s). In hydrodynamics, the units of a fluid's dynamic (shear) viscosity are $(\mathrm{N} \cdot \mathrm{s}) / \mathrm{m}^{2}=\mathrm{Pa} \cdot \mathrm{s}$. Thus, the shear stress $\tau$ can be interpreted as the rate of change of momentum $p$ per unit area $A$ (rate of momentum flux) of an arbitrary control surface as follows:

$$
\tau=\frac{p}{A}=\frac{m u_{x}}{A},
$$


where $u_{x}$ is the component of the average velocity of the molecules flowing parallel to $x$ plane through the unit area that is perpendicular to it, and $m$ is the mass of fluid flowing through the surface per unit time.

For a Newtonian fluid, when the stress is parallel to the surface, the shear stress $\tau$ per unit area is proportional to the rate of change of the velocity with respect to the distance and is represented as follows:

$$
\tau=\mu \frac{\mathrm{d} u_{x}}{\mathrm{~d} y},
$$

where $\tau=F / A$ and $\mathrm{d} u_{x} / \mathrm{d} y$ is the local shear velocity for a unit area parallel to the $x-Z$ plane, moving along the $x$-axis. The proportionality factor $\mu$ in this formula known as the dynamic viscosity. Therefore, the shear stress is the dynamic viscosity per unit area and has units of $(\mathrm{N} \cdot \mathrm{s}) / \mathrm{m}^{2}$.

Since the magnetic constant's units are N/A ${ }^{2}$, this indicates that it can be expressed as the shear stress per unit time or the dynamic viscosity per unit area and time. Therefore, an electron rotating in a vacuum experiences shear stress that diminishes its momentum, and this is equal to the magnetic constant. The shear stress depends on the vacuum's viscosity, which is related to its density. Therefore, the magnetic permeability is the rate of change of momentum per unit area and time that the electron encounters when rotating around its axis in a vacuum.

\section{Conclusions}

This paper has proposed a new interpretation of the essence and origin of the magnetic constant. An electron is considered as a vortex rotating in a superfluid vacuum, and the interaction length of the electron is equal to the Compton wavelength. Calculating diminished momentum of the rotating electron yields a value in the same range as the accepted magnetic constant value.

This result strongly suggests that the magnetic constant is related to the structure and properties of a physical vacuum, a medium that can be characterized by specific properties such as density, viscosity, and speed. Therefore, the magnetic constant is not a fundamental constant, but it is the diminished momentum of a rotating electron, which depends on the vacuum density. Its momentum per unit area is equivalent to its dynamic viscosity, which has units of $(\mathrm{N} \cdot \mathrm{s}) / \mathrm{m}^{2}$, while the rate of change of its momentum per unit area and time gives the change in the electron's dynamic viscosity per unit area and time. It is an expression of the force per unit area, acting parallel to an infinitesimal surface element due to vacuum viscosity.

This study opens up a new approach to determine the nature and essence of the fundamental constants of nature, related to vacuum density and thus to reduce the number of fundamental constants to one. The origin and essence of gravitation constant $\mathrm{G}$, fine structure constants was published in previous papers [1] [2], electric constant $\varepsilon_{o}$ speed of light $c$ constant and Planck constant will be presented in separate papers in the near future. 


\section{Limitations}

The superfluid nature of the vacuum still needs to be confirmed experimentally. In addition, even though the idea of electrons having a vortex structure is not new, it must still be confirmed by experimental research.

\section{Acknowledgements}

The author would like to thank Enago (https://www.enago.com/) for the English language review.

This research did not receive any specific grant from funding agencies in the public, commercial, or not-for-profit sectors.

Correspondence and requests for materials should be addressed to nader.butto@gmail.com.

\section{Conflicts of Interest}

The author declares no conflicts of interest regarding the publication of this paper.

\section{References}

[1] Butto, N. (2020) A New Theory on the Origin and Nature of the Fine Structure Constant. Journal of High Energy Physics, Gravitation and Cosmology, 6, 579-589. https://doi.org/10.4236/jhepgc.2020.64039

[2] Butto, N. (2020) New Mechanism and Analytical Formula for Understanding the Gravity Constant G. Journal of High Energy Physics, Gravitation and Cosmology, 6, 357-367. https://doi.org/10.4236/jhepgc.2020.63029

[3] Panofsky, W.K.H. and Phillips, M. (1962) Classical Electricity and Magnetism. Addison-Wesley, Reading, MA, 182.

[4] National Institute of Standards and Technology (2006) Introduction to the Constants for Nonexperts. Fundamental Physical Constants. Committee on Data for Science and Technology.

[5] Weiglhofer, W.S. (2003) The Classical Vacuum as Reference Medium. In: Weiglhofer, W.S. and Lakhtakia, A., Eds., Introduction to Complex Mediums for Optics and Electromagnetics. SPIE Press, Bellingham, WA, 34.

[6] National Institute of Standards and Technology (2006) Magnetic Constant. Fundamental Physical Constants. Committee on Data for Science and Technology. https://physics.nist.gov/cgi-bin/cuu/Value?mu0

[7] Rosen, J. (2004) Permeability (Physics) Encyclopedia of Physics. Facts on File Science Library. Facts on File, New York.

[8] Maxwell, J. (1873) A Treatise on Electricity and Magnetism. Sections 822 and 823.

[9] Butto, N. (2020) New Theory to Understand the Mechanism of Gravitation. Journal of High Energy Physics, Gravitation and Cosmology, 6, 462-472. https://doi.org/10.4236/jhepgc.2020.63036

[10] Sbitnev, V.I. and Fedi, M. (2017) Superfuid Quantum Space and Evolution of the Universe. In: Capistrano, A., Ed., Cosmology. InTech, Rijeka.

https://doi.org/10.5772/68113

[11] Sbitnev, V.I. (2016) Hydrodynamics of the Physical Vacuum: I. Scalar Quantum Sector. Foundations of Physics, 46, 606-619. https://doi.org/10.1007/s10701-015-9980-8 
[12] Sbitnev, V.I. (2016) Dark Matter Is a Manifestation of the Vacuum Bose-Einstein Condensate. http://arxiv.org/abs/1601.04536

[13] Sbitnev, V.I. (2016) Hydrodynamics of the Physical Vacuum: II. Vorticity Dynamics. Foundations of Physics, 46, 1238-1252. http://rdcu.be/kdon https://doi.org/10.1007/s10701-015-9985-3

[14] Zloshchastiev K.G. (2011) Spontaneous Symmetry Breaking and Mass Generation as Built-In Phenomena in Logarithmic Nonlinear Quantum Theory. Acta Physica Polonica B, 42, 261-292.

[15] Avdeenkov, A.V. and Zloshchastiev, K.G. (2011) Quantum Bose liquids with Logarithmic Nonlinearity: Self-Sustainability and Emergence of Spatial Extent. Journal of Physics B: Atomic, Molecular and Optical Physics, 44, 195303. https://doi.org/10.1088/0953-4075/44/19/195303

[16] Hubble, E. (1929) A Relation between Distance and Radial Velocity among Extra-Galactic Nebulae. Proceedings of the National Academy of Sciences of the United States of America, 15, 168-173. https://doi.org/10.1073/pnas.15.3.168

[17] Oldershaw, R.L. (1987) The Self-Similar Cosmological Paradigm: A New Test and Two New Predictions. Astrophysical Journal, 322, 34-36. https://doi.org/10.1086/165699

[18] Bonvin, V., et al. (2017) HoLiCOW V. New COSMOGRAIL Time Delays of HE0435-1223: $\mathrm{H}_{0}$ to $3.8 \%$ Precision from Strong Lensing in a Flat $\Lambda$ CDM Model Monthly Notices of the Royal Astronomical Society, 465, 4914-4930. https://doi.org/10.1093/mnras/stw3006

[19] Rauscher, E.A. (1968) Electron Interactions and Quantum Plasma Physics. Journal of Plasma Physics, 2, 517-541. https://doi.org/10.1017/S0022377800004013

[20] Rauscher, E.A. (2004) Dynamic Plasma Excitation Modes of Propagation in the Ionosphere. PA Press, Wisconsin, 295.

[21] The NIST Reference on Fundamental Physical Constants. https://physics.nist.gov/

[22] Helmholtz, H. (1858) Ueber Integrale der hydrodynamischen Gleichungen.

[23] Maxwell, J.C. (1861) XXV. On Physical Lines of Force, The Theory of Molecular Vortices Applied to Magnetic Phenomena. The London, Edinburgh, and Dublin Philosophical Magazine and Journal of Science, 21, 161-175. https://doi.org/10.1080/14786446108643033

[24] Butto, N. (2020) Electron Shape and Structure: A New Vortex Theory. Journal of High Energy Physics, Gravitation and Cosmology, 6, 340-352. https://doi.org/10.4236/jhepgc.2020.63027

[25] Butto, N. (2020) A New Theory on Electron Wave-Particle Duality. Journal of High Energy Physics, Gravitation and Cosmology, 6, 567-578. https://doi.org/10.4236/jhepgc.2020.64038

[26] CODATA 2014 Value for Compton Wavelength for the Electron from NIST. 